NOTAS Y COMENTARIOS

\title{
INTRODUCCIÓN A LA ECONOMÍA DE COMUNIÓNo
}

\author{
INTRODUCTION TO THE ECONOMY OF COMMUNION
}

Leonardo A. Caravaggio*

enviado: 2 mayo 2017 - aceptado: 22 junio 2017

\begin{abstract}
Resumen
Este trabajo se propone presentar una racionalidad económica alternativa llamada Economía de Comunión. Se trata de una idea fuerza que invita a participar de la actividad económica mirando, además del propio interés, el de quienes nos rodean. Hoy en día, más de ochocientas empresas en la Argentina y en el mundo adhieren a esta propuesta que tiene entre sus principales objetivos la eliminación de la pobreza.

Código JEL: D21, L21, M14.

Palabras clave: Economía de Comunión, economías alternativas, gestión de empresas.
\end{abstract}

\begin{abstract}
This work aims to present an alternative economic rationality called Economy of Communion. It is a leading idea that invites to participate in economic activity by looking, in addition to our own interest, to those around us. Today, more than eight hundred companies in Argentina and the world adhere to this proposal that has among its main objectives the elimination of poverty.

JEL Code: D21, L21, M14.

Keywords: Economy of Communion, Alternative Economies, Business Management.

- Caravaggio, L. A. (2018). Introducción a la economía de comunión. Estudios económicos, 35 (70), 99-109.

* Universidad de Buenos Aires. Correo electrónico: caravaggio@gmail.com
\end{abstract}




\section{LA HISTORIA DEL PROYECTO DE ECONOMÍA DE COMUNIÓN}

Luego del colapso económico soviético y la caída del muro de Berlín, la alternativa capitalista y el libre comercio se mostraban como el camino a seguir en términos económicos. Tras la crisis económica internacional del 2008, y las promesas incumplidas del modelo, surge la necesidad de una manera distinta de hacer economía. Así lo demuestra, por ejemplo, la expansión de las experiencias (especialmente en Europa) de la responsabilidad social empresaria (RSE) ${ }^{1}$. Esta iniciativa se ha desarrollado mucho y ha modificado ciertas formas del desarrollo económico. Sin embargo, muchas veces se ven carentes de un sustento teórico o un motivo más sólido por el cual ser socialmente responsables. Distinto es el caso de la propuesta económica que se intenta abordar en este trabajo, la Economía de Comunión ${ }^{2}$, la cual surge como expresión económica de una cultura distinta. Esta propuesta no solo es parte del desarrollo de esta cultura, sino que también da respuesta a la coyuntura económica actual, al menos así se puede interpretar su actual crecimiento y expansión. Se trata de una propuesta social y ambientalmente responsable que en teoría tiene la capacidad de generar un cambio cultural y redistribuir la riqueza. Implica la existencia de empresarios innovativos, al estilo schumpeteriano (Schumpeter, 1944), pero capaces de desarrollar las capacidades de las personas que los rodean, como aconsejaría Amartya Sen (1983, pp. 1115-1123).

El proyecto de EdC nace en el año 1991 de la mano de Chiara Lubich ${ }^{3}$ y en el seno del movimiento de los Focolares ${ }^{4}$. Si bien fue en ese momento presentado como una respuesta concreta al problema social de Brasil, el proyecto se extendió rápidamente por todo el mundo. $\mathrm{La} \mathrm{EdC}$ es una invitación a empresas ya existentes y a empresas por nacer a desarrollarse buscando el beneficio económico, pero también la "comunión" con las personas que rodean a la empresa, ocupándose especialmente de los más necesitados. El proyecto es revolucionario en la manera de entender la economía, ya que intenta desarrollar esta cultura desde la que nace, la "cultura del dar" (Quartana et al., 1992, pp. 31-32). Es una propuesta concreta para el modo de organización de las empresas que rápidamente consigue cientos de empresarios adherentes. Al cabo de un año del discurso con el que Chiara Lubich presenta la EdC ya se habían sumado al proyecto, principalmente en Brasil, doscientas treinta

1 Ver Resolución del Parlamento Europeo del 13 de marzo de 2007 sobre la responsabilidad social de las empresas: una nueva asociación. Disponible en www.europarl.europa.eu (acceso: mayo, 2017)

2 En adelante se hará referencia al término "Economía de Comunión" por su sigla EdC.

3 Fundadora del movimiento de los Focolares. Entre otras distinciones ha obtenido 15 doctorados honoris causa incluyendo el doctorado interdisciplinario de las 13 facultades de la Universidad de Buenos Aires.

4 Movimiento ecuménico presente en 182 países. www.focolare.org 
empresas y se había conformado un primer polo industrial a las afueras de la ciudad de San Pablo. Hoy en día existen más de ochocientas empresas adheridas al proyecto ${ }^{5}$, de los más diversos rubros y tamaños, presentes en los cinco continentes. Y ya son nueve los polos industriales. Si bien el proyecto es social y solidario, entiende perfectamente que la empresa debe ser económicamente rentable. Por lo tanto, se propone como objetivo ser autosustentable y que tenga además la capacidad de crecer, pero entendiendo que para crecer también es necesario que lo hagan los empleados de la empresa y toda la sociedad en la que la empresa está inserta.

La EdC es por naturaleza una economía popular, fraterna, de reciprocidad; no es, lo repetimos muchas veces, un proyecto dirigido solamente a los empresarios ni a los dirigentes, sino una propuesta vital destinada a todas las figuras de la vida económica, desde los trabajadores a los consumidores, para dar vida a nuevas organizaciones marcadas por la comunión. (Bruni y Calvo, 2008, p. 24).

A las empresas se les propone una tripartición de las ganancias, de modo que sean destinadas al crecimiento de la empresa, a ayudar a personas indigentes a salir de su situación y a la difusión de la cultura del dar, dando igual importancia a los tres objetivos (Lubich, 2007, p. 13). De este modo, el desarrollo de la sociedad y la justa distribución de la riqueza no se darían por medio de un estado centralizador del proceso económico, como en la experiencia comunista, ni tampoco por el libre juego del mercado, como propone el laissez faire, sino por la búsqueda de la comunión.

Como los más necesitados son el centro de la propuesta, muchas veces son los primeros en conseguir trabajo dentro de este tipo de empresas, o son apoyados para crear sus propios emprendimientos (Groppa y Sluga, 2015). Esto no se limita solamente a los pobres, sino que también existen empresas que se arriesgan y deciden contratar a quienes tienen necesidad del empleo aunque su capacitación al momento de tomar el cargo no se corresponda con las necesidades de la empresa, lo que puede implicar en principio mayores costos (Ruiz, 2014). Esta tripartición de las ganancias y la incorporación de los necesitados al proceso productivo permitieron, en la experiencia de Brasil, dentro del movimiento de los Focolares, la solución del problema de la pobreza. Con esta experiencia como modelo, tal vez sea solo cuestión de lograr una mayor adhesión al proyecto para resolver el problema a gran escala.

Con posterioridad al desarrollo empresarial, investigadores de distintos ámbitos de la ciencia se interesaron por este fenómeno que continuaba expandiéndose. Aparecen entonces los primeros desarrollos teóricos en los campos de la economía,

5 www.economiadecomunion.org (acceso: mayo, 2017) 
la sociología, la psicología, la ética, la administración de empresas y las relaciones del trabajo, entre otros. Hasta la actualidad, se ha publicado una amplia bibliografía traducida a varios idiomas, tanto específica de EdC (Quartana et al., 1992) como del marco teórico que la sustenta (Bruni, y Zamagni, 2009), y diversas tesis de graduación tanto de cursos de grado como de posgrado e incluso algunas a nivel de doctorado. Sin embargo, en Argentina el desarrollo teórico recién comienza; si bien muchos trabajos toman temas vinculados a esta nueva forma de hacer economía, pocos se dedican al proyecto con exclusividad.

\section{ECONOMÍA DE COMUNIÓN EN ARGENTINA}

El proyecto en Argentina comienza a dar los primeros pasos apenas dos meses después de su presentación en Brasil. El apoyo a la propuesta por parte de los adherentes al movimiento en Argentina es inmediato. Luego, unos meses después, con el aporte de cientos de adherentes, se logra comprar un terrero en O'Higgins, provincia de Buenos Aires, donde se pone en funcionamiento un polo industrial, uno de los primeros del mundo, al que se bautiza como "Polo Solidaridad".

En el Polo Solidaridad, hoy en día están radicadas siete empresas ${ }^{6}$, entre las cuales se encuentra UNIDESA SA (acrónimo de "Unidad y Desarrollo Sociedad Anónima"), que es la propietaria de las treinta y cuatro hectáreas de tierra donde funciona el polo. Esta empresa se gestó con un ideario muy inclusivo, con acciones de valor nominal bajo, para que cualquiera que quisiera pudiera participar con un pequeño capital. En 1995 se comenzó a construir la infraestructura para permitir a las personas que adhieren al proyecto vivir en el polo y así facilitar la instalación de las empresas en el lugar.

Con el tiempo se fueron edificando las calles, se realizó el tendido eléctrico, se construyó la red de agua potable (interna) y se contrató el servicio telefónico. Hoy ya hay varias casas construidas además de las empresas que allí funcionan. Sin embargo, todavía no resulta redituable económicamente para ninguna empresa establecerse en el polo. Las empresas lo hacen porque creen en el futuro del proyecto y porque ven el polo como una posibilidad de mostrar a otros esta nueva forma de hacer economía. En este sentido, las empresas allí radicadas no son las únicas beneficiarias del polo, sino, de algún modo, todas las que participan del proyecto de EdC.

6 Los datos de este punto fueron obtenidos de la página oficial de la EdC: www.edc-online.org 
El Polo Solidaridad funciona como difusor de la cultura y es la expresión más visible del proyecto en Argentina. Existe, por ejemplo, una exposición permanente ubicada en el Polo Solidaridad que recibe alrededor de tres mil visitantes por año, que llegan interesados por esta manifestación concreta del deseo de algunos empresarios por hacer una economía distinta.

Cada uno de los polos industriales de EdC conserva como rasgo particular las características del lugar donde está emplazado. En el caso del Polo Solidaridad, es marcadamente agrícola. Una de las empresas allí instaladas es la cultivadora Primicias, que cultiva seis hectáreas a cielo abierto, tres hectáreas bajo invernadero, y produce doscientas cincuenta toneladas de hortalizas y ochenta toneladas de verdura de hoja por año.

En todo el país la cantidad de empresas vinculadas con este tipo de economía asciende a más de ochenta, muchas de las cuales nacieron en adhesión al proyecto y otras han modificado su estructura preexistente para adaptarla a los términos de la Edc. Participan empresas de variados ámbitos y tamaños, tanto del sector primario como así también industrias y prestadoras de servicios. Entre los rubros representados, se encuentran estudios de arquitectura, consultoras económicas, estudios contables, restaurantes, explotaciones agropecuarias, producción de alimentos, criaderos de ganado, establecimientos de apicultura, imprentas, empresas de desinfección y control de plagas, establecimientos metalúrgicos. Los empresarios de EdC en Argentina se reúnen periódicamente para intercambiar experiencias y sostenerse en la adhesión al proyecto.

\section{UN MODELO DE GESTIÓN DIFERENTE}

La propuesta de EdC tiene como rasgo distintivo la distribución de ganancias que realizan las empresas. Sin embargo, esto no alcanza para describir acabadamente el proyecto, ya que la EdC propone también un modelo de gestión diferente de la empresa. Y más aún, el proyecto no concluye con las decisiones de la empresa y los empresarios, sino que es una propuesta vital para todos lo empleados. Esto quiere decir que la empresa en su totalidad es gestionada de una nueva forma: el empresario primero y todos después buscan en cada decisión la comunión. Esto es lo que lleva a la empresa a posteriori a ser socialmente responsable, a cuidar el medioambiente, a respetar a los empleados, a valorar los tiempos de trabajo, a mantenerse dentro del marco de la legalidad y a distribuir las ganancias. La propuesta no es generar ganancias "a como dé lugar" para después distribuirlas, ni tampoco "dar una imagen" de responsabilidad social. 
A continuación, se propone una tipología que puede servir para identificar las características de las empresas que buscan ser socialmente responsables (Bruni, y Calvo, 2009). En primer lugar, algunas empresas buscan ser socialmente responsables, no por iniciativa propia, sino bajo una presión impuesta por la sociedad y guiadas por intereses políticos o, incluso, por el mero cálculo económico, dado que ser socialmente responsables puede ser un costo ineludible para poder participar de determinados mercados. Este tipo de empresas actúan de esta manera solo en el contexto histórico geográfico en el que están insertas, y dejarían de hacerlo si la presión exterior menguara. Un segundo tipo de empresa es el que usa la RSE como un medio de marketing. Dado que la responsabilidad social es bien vista, responder a determinados criterios puede hacer a la empresa aumentar las ventas. En este caso, a diferencia del primero, el ser socialmente responsable es una elección libre. El problema de este tipo de empresas radica en que si no encuentran una respuesta por parte de los consumidores, dejarán de ser responsables. Por último, existe un tercer tipo de empresas, que son socialmente responsables por razones intrínsecas, es decir, por motivaciones internas. La empresa, esto es: sus dueños o sus gerentes, han interiorizado valores éticos que los llevan a esta decisión en libertad y sin depender de la respuesta de terceros.

Las empresas de EdC (no solo ellas) pertenecen a este tercer tipo. Es decir que hay un motivo más sólido por el cual ser socialmente responsables no es una regla impuesta, ni tampoco una cuestión de conveniencia económica (extrínseca), sino una elección libre e independiente. Esta elección es una vocación o una manera de pensar que lleva a este tipo de compañías a vincularse de una forma distinta con su contexto, poniendo siempre a la persona en el centro, y no a las ganancias, y que se refleja en todos los aspectos de la vida de la empresa. De esta manera las empresas de EdC optan por un estilo de gestión diferente, que no solo implica una redistribución de las ganancias sino un accionar distinto respecto de la empresa tradicional en todos los ambientes y en todas las decisiones que se toman.

En los encuentros que al menos anualmente tienen los empresarios con cada uno de sus empleados se observan los progresos y fracasos en cada uno de estos ambientes. Lo importante no es que la empresa logre sostenerse (a cualquier precio), sino que consiga mejorar la calidad de vida dentro y fuera de la organización. Queda así en evidencia la imposibilidad de los empresarios de llevar adelante los proyectos de manera aislada; por el contrario, aquí se busca siempre el consenso con los empleados, en una gestión mucho más democrática que en las empresas tradicionales.

El objetivo de la empresa —esto es, no solo obtener ganancias, sino también ayudar a los necesitados y expandir la cultura del dar- es llevado adelante por 
todos en la empresa, desde los gerentes hasta los empleados. En este sentido debe ser entendida la palabra "comunión" en el ámbito de la EdC: es una economía "con el otro", una economía donde "el otro" importa.

Como mencionamos, no se espera que por el solo hecho de maximizar ganancias las empresas sean socialmente eficientes - en el sentido de la mano invisible de Adam Smith-. Tampoco es suficiente con seguir una "lógica de los dos tiempos" - donde primero las empresas producen las ganancias y a posteriori el Estado, o ellas mismas, redistribuyen-. Por el contrario, las empresas de EdC deciden ser socialmente responsables durante el proceso de producción, en cada decisión que toma la empresa.

Es importante destacar el rol de la decisión libre en este proceso, nadie puede imponerle a la empresa tales conductas. Tan importante es la libertad de acción para el proyecto que muchas veces se hace referencia al mismo como "Economía de Comunión en Libertad".

Puede pensarse en ocho ámbitos en los que las empresas buscan desempeñarse responsablemente de modo de lograr ser creadoras de la cultura del dar y generadoras de mejoras para la sociedad (Ferrucci, 2013). A continuación, se hace una breve referencia a cada uno de estos ámbitos.

\section{Economía y trabajo}

Más allá de su fin social, las empresas de EdC son empresas con fines de lucro, y se proponen como objetivo una buena administración en lo económico que les permita sostenerse y crecer. Esto produce la primera ayuda a los necesitados, que no es el aporte económico que nace de la tercera parte de las ganancias, sino la posibilidad de brindarles trabajo que tiene la empresa.

El trabajo permite a los empleados sostener a su familia y salir de su situación de necesidad. En este sentido, las empresas de EdC adoptan incluso riesgos razonables, como colaboraciones económicas en momentos de dificultad, contrataciones de personas que tendrían problemas para insertarse en empresas tradicionales, entre otros. Esto tiene sentido ya que ayudar a los empleados y a la sociedad es un objetivo de la empresa en la misma medida que lo es la obtención de ganancias. De esta manera, se favorece también la adopción por parte de los empleados de los objetivos de la empresa. Es decir que en las empresas de EdC conviven los criterios económicos de maximización de ganancias y la convicción de que una sociedad mejor es posible. 
2. La relación con los clientes, los proveedores, la sociedad civil y los sujetos externos

Las empresas de EdC se proponen ofrecer bienes y servicios útiles a precios justos. En determinadas ocasiones, las empresas tradicionales pueden ofrecer bienes de mala calidad o impulsar por medio de publicidad compras innecesarias con el solo propósito de aumentar los beneficios.

Como para las empresas de EdC los beneficios no son el único objetivo, tiene sentido relegar la obtención de ganancias procurando no aprovecharse de los clientes, ni sacar ventajas de las asimetrías de información. De esta manera, buscan también relacionarse en forma leal con la competencia actuando con respeto recíproco. Así las relaciones con los clientes, proveedores y todas las personas ajenas a la empresa se convierten en un bien en sí mismo.

\section{3. Ética}

La ética interna de los empresarios de EdC es, en buena medida, la razón que impulsa a las compañías a adherir a esta propuesta. La ética lleva a las empresas a mantenerse dentro del marco de la legalidad imperante en su territorio, tanto en lo que respecta a las autoridades fiscales como a los organismos de control y los sindicatos, entre otros. Sería un contrasentido que el dinero que luego es redistribuido por la empresa proviniese de evasiones fiscales u otras acciones ilegales.

4. Salud y valor de la vida

Comenzando por los propios empleados y por cada miembro de la empresa, poner a la persona en el centro implica también preocuparse por su salud.

Las empresas de EdC buscan evitar que sus empleados se sobrecarguen con horarios de trabajo demasiado prolongados, como así también valoran el tiempo de vacaciones como una necesidad de descanso. Existen ciertos criterios de seguridad en el trabajo, que generalmente están regulados por las leyes de cada país, que se buscan respetar en todos los casos: ventilación necesaria, niveles tolerables de ruido, iluminación adecuada, vestimenta de seguridad, etcétera. El clima laboral y las relaciones fraternas dentro de la empresa también contribuyen a la salud y el valor por la vida. 
La preocupación por la salud de las personas externas a la empresa lleva también a repensar los bienes y servicios que se producen, ya sea por el daño directo que estos puedan provocar como así también los daños al medioambiente que terminan afectando la salud de la sociedad.

\section{Armonía en el ambiente de trabajo}

Las empresas de EdC se organizan internamente de forma lo más democrática posible. Aspiran de esta forma a favorecer el crecimiento de los empleados, tanto en tareas grupales como individuales. Los empleados suelen sentir a la empresa como propia y se esfuerzan por mantener el lugar de trabajo limpio y el ambiente agradable. Todos los empleados, al actuar de esta manera, la atención de la sociedad, los clientes y los proveedores y así transmitirles esta nueva cultura.

\section{Formación e instrucción}

Dado que las personas son consideradas el centro de la empresa, su desarrollo y crecimiento se convierte en uno de los objetivos de la misma. Es por esto que se intenta favorecer la formación e instrucción de cada una de las personas que trabaja en la empresa, ya sea permitiendo días de estudio solventando los costos de cursos u otros.

La ayuda se intenta brindar tanto en los casos en los que estudios tienen una relación directa con las necesidades de la empresa como en los casos en los que no. La formación de los empleados, en principio, se vincula con que lo que importa es la persona en sí misma, más allá de la función que desempeñe en la compañía; por otro lado, se considera que el crecimiento del empleado siempre es redituable para la empresa, ya que no se sabe cuándo los conocimientos aprendidos podrán serle útiles.

\section{Comunicación}

Otro de los objetivos de la empresa de EdC es el de crear un clima que favorezca una comunicación abierta y sincera entre los miembros, que permita el intercambio de ideas entre los dirigentes y los trabajadores. 
También se busca permanentemente la comunicación con otras empresas de EdC, manteniéndose en contacto, especialmente las vinculadas por rubro, o reuniéndose periódicamente para compartir las experiencias de la adhesión al proyecto.

\section{La gestión de la empresa}

Los responsables de las empresas de EdC buscan formular estrategias, objetivos y proyectos junto a los demás miembros, recurriendo para esto a técnicas modernas de gerenciamiento orientadas a la persona.

Dentro de la empresa los miembros intentan mantener un clima de respeto, confianza y apoyo recíproco. Cuando la "cultura del dar" logra expandirse, los miembros de la empresa no se la guardan para sí, sino que ponen libremente a disposición sus talentos, ideas y capacidades a favor del crecimiento profesional de sus colegas y para el progreso de la empresa. Con el objetivo de verificar que todos estos objetivos se estén llevando adelante, y comprobar la calidad de las relaciones interpersonales, tanto empresarios como empleados procuran encontrarse regularmente. De esta manera, la colaboración de cada uno de los miembros de la empresa al enfrentar las dificultades y adversidades es muy importante.

\section{REFERENCIAS BIBLIOGRÁFICAS}

Bruni, L., \& Calvo, C. (2008). El precio de la gratuidad: Nuevos horizontes en la práctica económica. Buenos Aires: Editorial Ciudad Nueva.

Bruni, L., \& Zamagni, S. (comp.) (2009). Persona y comunión. Buenos Aires: Editorial Ciudad Nueva.

Ferrucci, A. (2013). Líneas para dirigir una empresa de Economía de Comunión. Recuperado de http://www.edc-online.org/es/empresas/lineas-para-dirigir-la-empresa.html.

Groppa, O., \& Sluga, M. L. M. (2015). Empresas y bien común: caracterización de las empresas de economía de comunión y empresas B en la Argentina. Revista Cultura Económica, 33 (89), 8-24.

Lubich, C. (2007). Economía de comunión: Historia y profecía. Buenos Aires: Editorial Ciudad Nueva.

Quartana, P., Sorgi, T., Araujo, V., Gui, B., \& Ferrucci, A. (1992). Economía de Comunión: Propuestas y reflexiones para una cultura del dar. Buenos Aires: Editorial Ciudad Nueva. 
Ruiz, J. (2014). Otra racionalidad empresaria: casos de empresas argentinas. Manuscrito inédito, Centro de Estudios de la Situación y Perspectivas de la Argentina, Universidad de Buenos Aires, Argentina.

Schumpeter, J. A. (1944). Teoría del desenvolvimiento económico. Una investigación sobre ganancias, capital, crédito, interés y ciclo económico. México DF: Fondo de Cultura Económica.

Sen, A. (1983). Los bienes y la gente. Revista Comercio Exterior, 33 (12), 1115 1123.

(C) 2018 por los autores; licencia otorgada a la revista Estudios económicos. Este artículo es de acceso abierto y distribuido bajo los términos y condiciones de una licencia Atribución-No Comercial 3.0 Unported (CC BY-NC 3.0) de Creative Commons. Para ver una copia de esta licencia, visite http://creativecommons.org/ licenses/by-nc/3.0 
